\title{
Focus on rib fracture fixation after trauma
}

\author{
Luke P. H. Leenen ${ }^{1}$
}

Published online: 26 July 2019

(c) Springer-Verlag GmbH Germany, part of Springer Nature 2019

\section{Dear colleagues,}

A large part of the articles in this focus on thoracic injury is dedicated to thoracic wall injuries. We have seen over the last years a renewed interest in rib fixation, because new methods have become available to repair rib fractures, but more and more evidence has also become available revealing that rib fractures, especially in older patients, is not an innocent injury. However, the quest for the right indication for the right patient is on!

That age is an important factor has been substantiated in a study from Rotterdam, the Netherlands [1]. Dr Vledder and his team described the pattern of injuries and outcomes in the elderly patients. They conclude that age plays an important role in mortality as the presence of several co-morbidities. Additionally, injuries in different body regions also play an important role. In summary, pneumonia and death occur in approximately $10 \%$ of elderly patients with substantial chest injury, which stands out from the average trauma patient.

Chest injury and not only the chest wall is a major injury. This was shown by Heinanen et al. who evaluated the resource use as well as outcome in a 10-year registry study [2]. They also made a comparison between Finland and Germany for pre- and in-hospital resource use. Interestingly, major differences were found in pre-hospital resources as well as methodology, resulting in also higher resource use in hospital. However, surprisingly, no differences in the outcome were found.

As pain is an important aspect for the treatment of rib fractures, analgesic intervention has been a long-standing accepted treatment modality for rib fractures. Until now, there has not been a comprehensive overview of the different techniques used to relieve the burden of pain in these patients. Peek et al. evaluated in a systematic review and

Luke P. H. Leenen

1.p.h.leenen@umcutrecht.nl

1 Department of Trauma, University Medical Centre Utrecht, Utrecht, The Netherlands meta-analysis, the contribution of several analgesic interventions and their respective success [3]. They conclude that there still is no single hammer to hit the nail in this respect, however, that until now, epidural analgesia seems to be the most effective treatment, when successfully applied.

Another interesting study in this respect was presented from Germany (Akil et al.), where the pain-killing effect of surgical SIB stabilization has been detailed [4]. In this day and age of an opioid crisis (at least in the States) and from the perspective of the trauma surgeon, this is an interesting issue. They conclude that rib stabilization of unstable rib fractures after blunt trauma is a safe and effective treatment option that leads to a significant reduction in trauma-associated pain, supporting a quick recovery. These successes in individual patients, as presented in this study, drive believers of rib fixation into the active "fixation" mode. Still, however, a definitive answer whether rib fixation can be unequivocally supported for every rib fracture has still not been given.

Based on this observation, in another meta-analysis and systematic review, Beks et al. summarized the current evidence for fixation of rib fractures and also tried to find some future guidance [5]. They concluded after evaluating 33 studies with over 5800 patients that for flail chest there is indeed a short-term improvement proven in literature, however, that for multiple rib fractures the answer is still out.

As short-term results in many studies show favorable results, there is only scarce information of the long-term results of fixation of rib fractures. In a long-term follow-up study by Beks et al., patient-reported outcomes were also studied in patients several years after rib fixation [6]. They show that rib fixation is a safe procedure and that patients reported a relative good quality of life. Patients should be counseled that after rib fixation, approximately half of the patients will experience implant-related irritation and about one in ten patients requires implant material removal.

Nevertheless, the ultimate proof of the pudding is the question whether rib stabilization is better than non-operative treatment. This is presented in the last article of the focus on this issue. Beks et al. compared two large level 1 trauma centers in the Netherlands with each other [7]. One 
center is doing surgical rib fixation as a routine, whereas in the other hospital, surgical rib stabilization never takes place. In this "geographically randomized" study, two cohorts were propensity matched and compared. The surprising results show that there is no advantage for either arm of the study. This means that up to now, there still is no definitive answer. Probably sorting out the right patient population that unambiguously benefits from surgical stabilization is still not clear.

Further studies are warranted to find this holy grail of chest wall surgery.

Dear readers, again this is a challenging focus on issue on thoracic trauma. The field is really on the move and a lot of studies try to reveal different aspects of our daily care to improve treatment of the patient with injuries to the chest. These articles again will support your daily practice and move it up to the current cutting edge of trauma surgery.

\section{Compliance with ethical standards}

Conflict of interest The author has a grant from Dupuy Synthes for a prospective observational cohort study on rib fractures.

\section{References}

1. Van Vledder MG, Kwakernaak V, Hagenaars T, Van Lieshout EMM, Verhofstad MHJ, On behalf of the South West Netherlands
Trauma Region Study Group. Patterns of injury and outcomes in the elderly patient with rib fractures: a multicenter observational study. Eur J Trauma Emerg Surg. 2018. https://doi.org/10.1007/ s00068-018-0969-9.

2. Heinänen M, Brinck T, Lefering R, Handolin L, Söderlund T. Resource use and clinical outcomes in blunt thoracic injury: a 10-year trauma registry comparison between southern Finland and Germany. Eur J Trauma Emerg Surg. 2018. https://doi. org/10.1007/s00068-018-1004-X.

3. Peek J, Smeeing DPJ, Hietbrink F, Houwert RM, Marsman M, de Jong MB. Comparison of analgesic interventions for traumatic rib fractures: a systematic review and meta-analysis. Eur J Trauma Emerg Surg. 2018. https://doi.org/10.1007/s00068-018-0918-7.

4. Ali A, Stephan Z, Jan R, Michael S, Christian MM, Stefan F. Rib osteosynthesis is a safe and effective treatment and leads to a significant reduction of trauma associated pain. Eur J Trauma Emerg Surg. 2019. https://doi.org/10.1007/s00068-018-01062-5.

5. Beks RB, Peek J, de Jong MB, Wessem KJP, Öner CF, Hietbrink F, Leenen LPH, Groenwold RHH, Houwert RM. Fixation of flail chest or multiple rib fractures: current evidence and how to proceed. A systematic review and meta-analysis. Eur J Trauma Emerg Surg. 2018. https://doi.org/10.1007/s00068-018-1020-x.

6. Beks Reinier B, de Jong MB, Houwert RM, Sweet AAR, De Bruin IGJM, Govaert GAM, Wessem KJP, Simmermacher RKJ, Hietbrink F, Groenwold RHH, Leenen LPH. Long-term followup after rib fixation for flail chest and multiple rib fractures. Eur J Trauma Emerg Surg. 2018. https://doi.org/10.1007/s0006 8-018-1009-5.

7. Beks RB, Reetz D, de Jong MB, Groenwold RHH, Hietbrink F, Edwards MJR, Leenen LPH, Houwert RM, Frölke JPM. Rib fixation versus non-operative treatment for flail chest and multiple rib fractures after blunt thoracic trauma: a multicenter cohort study. Eur J Trauma Emerg Surg. 2018. https://doi.org/10.1007/s0006 8-018-1037-1. 